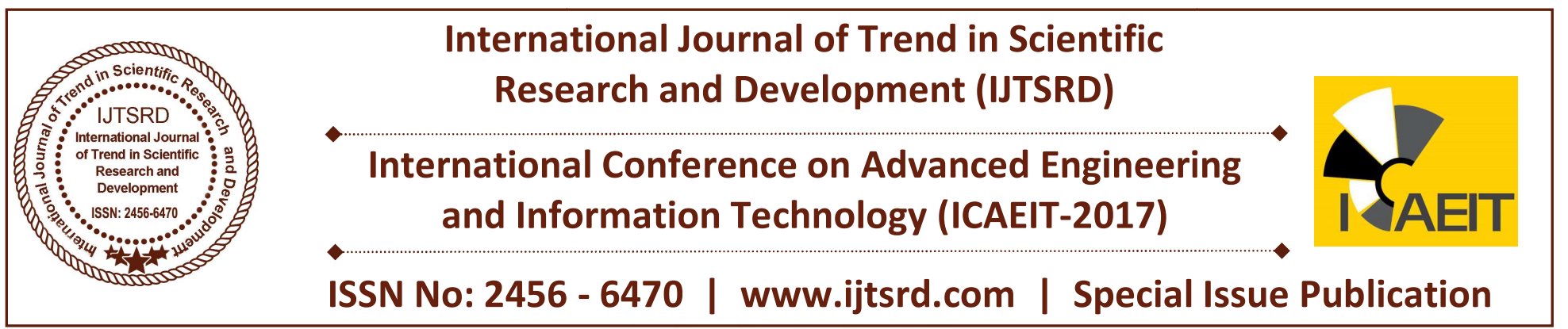

\title{
Quality Factor between Visual Studio and Android Studio for Developing Video Control Based on Hand Gestures
}

\author{
Siti Norhidayah Aman, Noor Zuhaili Md Yasin, Illiana Azizan \\ Faculty of Engineering and Technology, Linton University College, Negeri Sembilan, Malaysia
}

\begin{abstract}
In this paper, research of a video control, based on hand gesture which recognizes a play and pause, is proposed. In the field of image processing, being able to recognize human gesture in daily life application is one of the most appealing technology as it represents the communication between human and machine. Comparative tools such as Visual Studio (VS) and Android Studio (AS) can be used in developing the video control based on hand gestures. A comparison that will be discussed in this paper is based on the quality factor which is the simplicity for each of the software proposed.
\end{abstract}

Keywords: Video Control, Hand Gesture, Visual Studio (VS), Android Studio (AS), Quality Factor

\section{INTRODUCTION}

Hand gesture apparently need more advanced technology in order for the system that applied the function to run smoothly. Gesture recognition is defined as a "mathematical interpretation of a human motion by computing device" [10] and image processing "is the study of any algorithm that takes an images as input and return an image as an output" [2]. However, with current software technology it is only possible to make it look nice and more significantly, the system also can perform based on the required functions such as play and pause. Non-functional requirement is specified "what must behave" [12] for the system. As non-functional requirement for the simplicity, it can be understood as how the system is "easy to understand or explain" [12]. It is clear that Visual Studio have a myriad of function that can be used as it is not restricted only for the form development but also web, windows presentation foundation (wpf) application and android development. Moreover, based on the software name itself indicates that Android Studio is used for android development purpose only.

\section{OVERVIEW OF THE TOOLS}

Microsoft Visual Studio (VS) is "an integrated development environment (IDE) from Microsoft. It is used to make app development easy for any platform and language" [3]. VS enable users to write coding "accurately and efficiently without losing current file context" [3]. The functionality also can be control into "refactor, identify and fix code issues" [3].

While, Android Studio (AS) is an "official IDE for android's" [4] and have the purpose to build for android application in order to "accelerate the development and help developer to build the highest quality apps for each and every android device" [4]. This tool offers the developer the functionality of "rich code editing, debugging, testing and profiling tools" [4].

\section{SIMPLICITY}

"Quality that is identified independent of any measurable characteristics. That is, quality is defined as the products or services capability to meet customer expectations" [13]. To ensure the quality of product, quality factor will represent the behavioral characteristic of system. The quality factors or nonfunctional requirement describe "different types of system behavior characteristics, and the quality criterions are attributes to one or more of the quality factors" [15]. 
Quality model is "the represent of the characteristics of the software that describe the quality factor" [15]. McCall's quality model [11] has figured "between users and developers by focusing on a number of software quality factors that reflect the users' perceptions and the developers' priorities" [14]. However, the problem in this model is "no method, no standard and tool to measure these quality factor" [11].

Jim McCall et al [7] has identified 11 quality factors in order to ensure the quality of product to meet user satisfaction. One of 11 quality attribute is simplicity. Simplicity can be defined as the ease with which software can be understood by the user. The quality factor is important in order to obtain to view of quality based on users or customer perspective.

As human hand is a complex object that required a myriad of devices in order to recognize and tracked the image, the implementation of hand gesture is still in a research development. Even if it the implementation occurs, it still limited to a specific application such as music control and PowerPoint slide control. In awareness of this matter, the implementation of hand gesture recognition also new to the normal user. This indicates that the system should be develop as a simple as possible with the functions that a normal user can understand how to using it.

Hayim Makabee has written a good article about simplicity in software design. The author says:

"A simplistic software design may be one that focuses only on present requirements and ignores future needs such as maintainability, extensibility and reusability." [1].

The author also added that:

"I think that the danger of simplistic design is very well expressed by this quote:

'Keep it simple, as simple as possible, but not simpler.'

- Albert Einstein" [1]

Based on the above statement, the simplicity can be applied to the hand gesture system to the maximum as long as the features of the system is fully function and all user from normal ones to the professional user such as IT user can understand easily on how to used it. This is important for the system to be introducing worldwide before it can be upgrade and used daily in the future.

In Visual Studio, the video control based on hand gesture system has the simplicity in creating the Graphical User Interface (GUI). "A GUI framework allows user to create an application with a wide range of GUI elements such as labels, textboxes and other well-known elements" [5]. Without this framework, the developer will have to draw "these elements manually and handle all the work of the user interactions scenario such as text and mouse input" [5]. Instead of making a lot of work, GUI framework is used by most of developers as the framework "will do all the basic work and allowed them to focus on making great applications" [5].

As long as the system can play the video, the interface of the system should be simple enough as the main idea of the system is to play and pause using hand gestures. The system is used the GUI framework in order to create the simple interface. The focus of the system interface is how the video will be played and paused easily. The system is create with only have "Open" file for user to find the movie included play and pause button for starter.

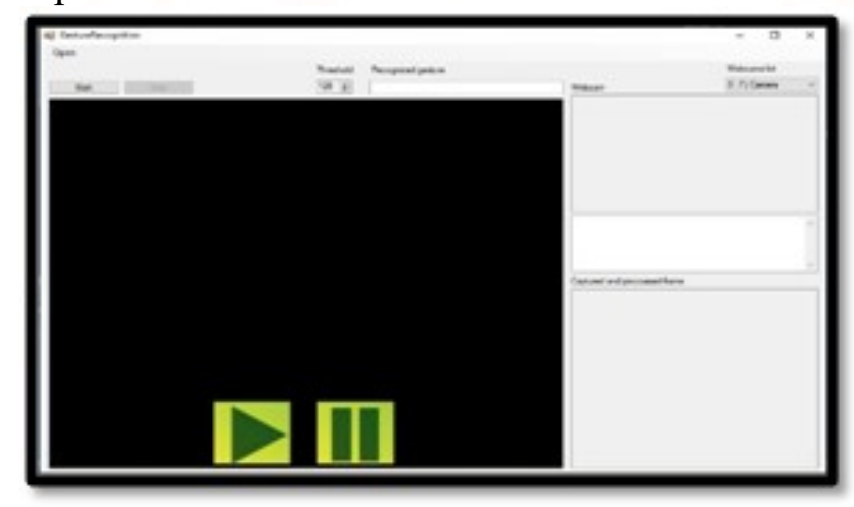

Figure 1: Interface design of the system based on simplicity

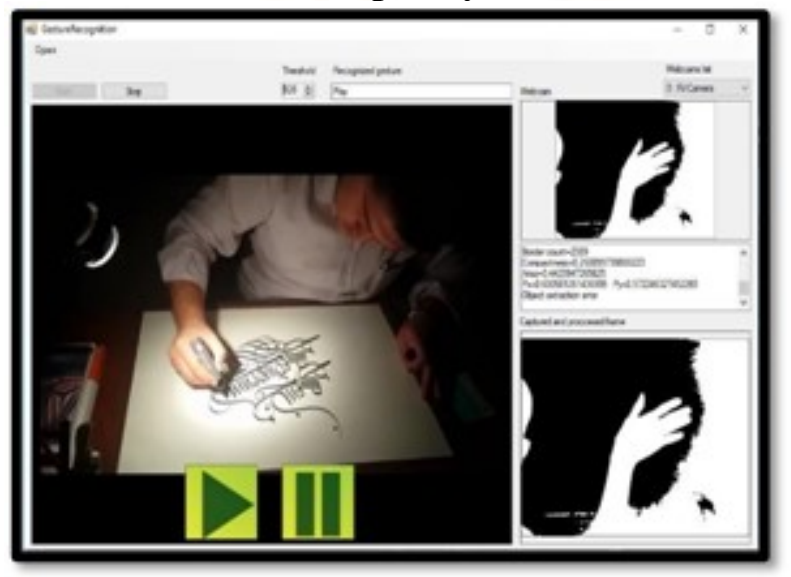

Figure 2: The system is worked by played the video 


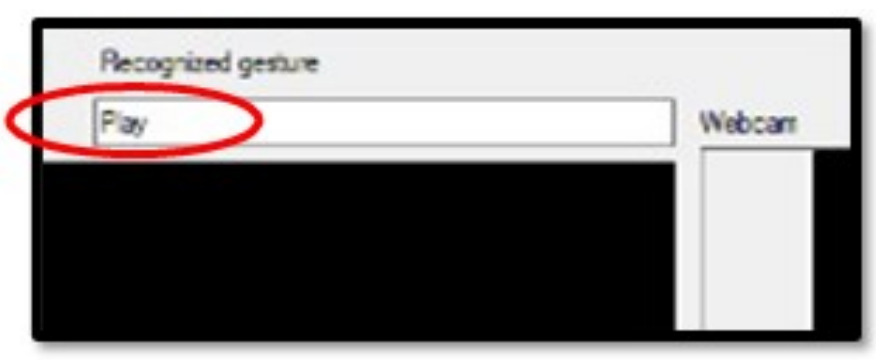

Figure 3: Play gesture is recognized

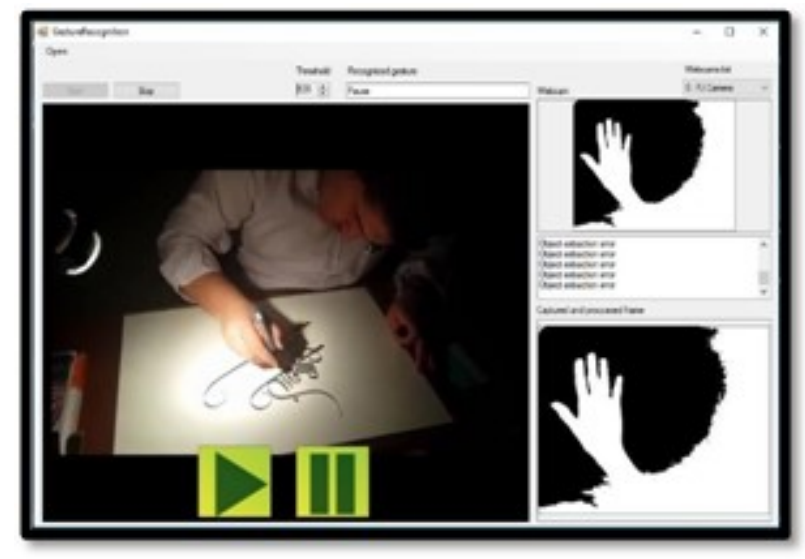

Figure 4: The system is worked by paused the video

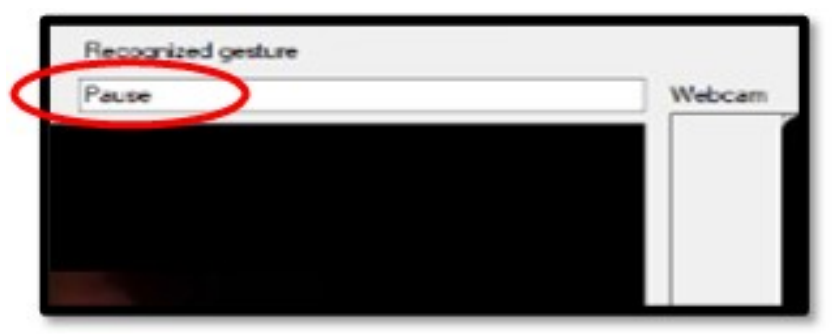

Figure 5: Pause gesture is recognized

On the other hand, Android Studio simplicity is based on feature such as "development for all Android devices" [4]. That is to say, Android Studio offers multiple environment using a single project to developing the application. These application can have a sharing code among different version of app such as Android phones, Android TV, tablets, Android Wear and Android Auto.

In addition, Android Studio simplicity feature also implied to the "robust and flexible build system" [4] for the application. Based on Jagdish L. Raheja researched, for the portable hand sign recognition to be able to used, "computer vision based techniques were used for image analysis and PCA was used after image tokenizer for recognition. This method was tested with webcam results to make system more robust" [8].
Following this further, the best example is the android SDK. With android SDK, developer can create their own applications or changing the platform. Based on Matthias Mellouli, "this kit contains a set of libraries, powerful tools for debugging and development, a phone emulator, thorough documentation, FAQ and tutorials" [9]. For instance, high performance build automation can be performed using Gradle and resulted to the developer can easily plug all the holes of the system when it crashes.

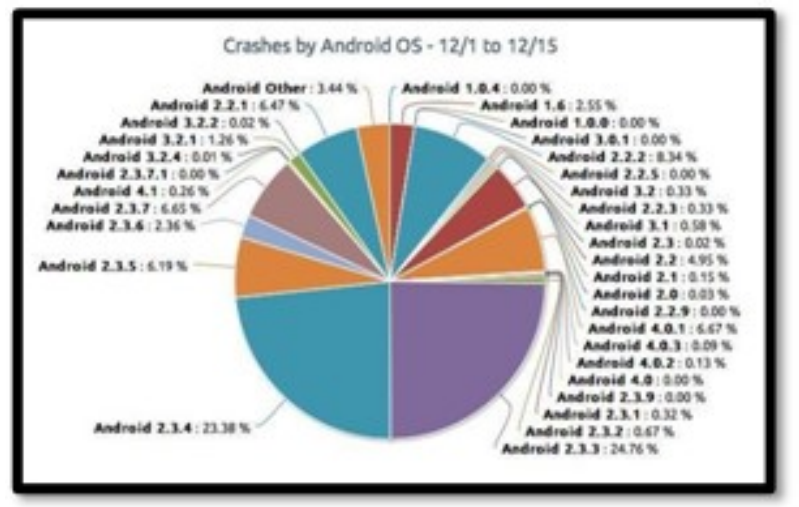

Figure 6: Statistic crashes by Android OS [6]

\section{FUTURE ENHANCEMENT}

Gesture recognition is a new advance technology that is important these days as it is most likely will play a major role in human daily lives in the future. The hand gesture can be used in advance to control applications and enter new area besides than media player such as browser, internet of things (IoT), medical area and army area. Certainly, a simple way to have better lives. However, for the implementation to be applied, the system must be simple enough for everyone to be using it.

On the contrary, this review is one of the work that has been done in order to achieved the objectives of creating the video control based on hand gesture using the best tools. In order that, the non-functionality requirement which is simplicity is chosen to compare these tools that is Visual Studio and Android Studio. Though this review is presented based on the analysis and critical thinking through the research done, it is possible that it will have a mistake. Before, this review is only based on research. But, when the system is ready in full functionality using both of tools, this review will be fixed in the future work.

\section{CONCLUSION}

The quality factor presented within this paper by using different tools probably "could be fitted within the user view, manufacturing view or product view" 
[15]. However, the advantages are the quality factor that I has choose to reduce "the notion of quality to a few relatively simple and static attributes" [15]. The benefit of this quality factor is that they are simpler to use and easy to understand. The good of quality factor are depending on the good quality model used to ensure the product has meet user satisfaction. Some "quality models such as Boehm, FURPS, Dromey and ISO 9126 also has its own quality characteristics along with the factors and sub-factors" [15].

In final analysis, video control based on hand gesture provided its own versatile form by using either Visual Studio or Android Studio. The used term simplicity in both software is such contrary as Visual Studio provide simplicity in graphical user interface (GUI) which make it easy to focus on developed a good application without worried the small details. The quality factor of simplicity is achieved as shown in the images above. In contrast, the term simplicity for Android Studio is the features that is given to it. Hence, the development of video control based on hand gestures by using visual studio with efficient software quality factor in that will assist in the creation of high quality software will become one of the most important research area in my future works.

\section{ACKNOWLEDGEMENT}

The authors would like to express their gratitude to all the members of the Faculty of Engineering and Technology of Linton University College for all the detailed and helpful suggestions and comments.

\section{REFERENCES}

1. Hayim Makabee, "Simplicity in Software Design: KISS, YAGNI and Occam's razor", 2013

2. "Introduction: What is image processing?" from http://www.coe.utah.edu/ cs4640/slides/Lecture0. pdf, 2012

3. Microsoft, "Visual Studio IDE: Build apps of the future today", from https://www.visualstudio.com/,2017
4. "Android Studio: The Official IDE for Android", from

https://developer.android.com/studio/index.html 2017

5. "What is WPF?" from http://www.wpftutorial.com/about-wpf/what-is-wpf/, 2012-2017

6. Tomio Geron, "Do iOS Apps Crash More Than Android Apps? A Data Dive", 2012

7. Ines Fey, DaimlerChrysler AG, Berlin, Germany. Ingo Sturmer, Model Engineering Solutions, Berlin, Germany, "Quality Assurance Methods for Model- based Development: A Survey and Assessment", SAE International, 2007.

8. Jagdish L. Raheja, A. Singhal, Sadab, "Android based Portable Hand Sign Recognition System", 2015.

9. Matthias Mellouli, "PROJECT REPORT Android Application”, 2010.

10. Margaret Rouse, "Gesture recognition" from http://whatis.techtarget.com/definition/gesturerecognition, 2015.

11. Rikard Edgren, "Software Quality Models and Philosophies", 2010.

12. Ramesh Kartik, "Non-Functional Requirements (NFR) in Agile Practices", 2013.

13. "Chapter_One: 1. Software Quality Models and Philosophies" from http://www.bth.se/com/besq.nsf/ (WebFiles)/CF1C3230DB425EDCC12570690031 7C 44/\$FILE/chapter_1.pdf, [no date].

14. Singh, B. and S. P. Kannojia, "A Review on Software Quality Models", International Conference on Communication Systems and Network Technologies, 2013

15. Noor Zuhaili Md Yasin, "Software Quality Factor and System Overview", 2014. 